\title{
Role of cGMP dependent protein kinases in the circadian system Urs Albrecht* ${ }^{*}$, Sonja Langmesser ${ }^{1}$, Henrik Oster ${ }^{1}$, Franz Hoffmann ${ }^{2}$ and Robert Feil ${ }^{2}$
}

Address: ${ }^{1}$ Universität Fribourg, Department of Biochemistry, Fribourg, Switzerland and ${ }^{2}$ Institut für Pharmakologie und Toxikologie, TU München, Germany

Email: Urs Albrecht* - Urs.albrecht@unifr.ch

* Corresponding author

from 2nd International Conference of cGMP Generators, Effectors and Therapeutic Implications

Potsdam, Germany, 10-12 June, 2005

Published: 16 June 2005

BMC Pharmacology 2005, 5(Suppl I):S6 doi:I0.|I86/I47|-22 I0-5-SI-S6

Several signal transduction pathways are involved in the resetting mechanism of the circadian clock. A role of cyclic GMP in this mechanism has been suggested. Pharmacological agents can upregulate cGMP and activate cGMPdependent protein kinases (cGKs also known as PKGs) of the suprachiasmatic nuclei (SCN) in brain slice cultures. In mammals both cGK isoforms, cGKI and cGKII are expressed in the SCN indicating a role for these kinases in the resetting mechanism of the circadian clock. We present genetic evidence for these kinases that they play a role in this mechanism in vivo. For cGKI mutant mice it takes more time to adapt to new lighting conditions than for wild type animals but they finally adapt to a jet-lag. Interestingly, mice mutant in cGKII can not delay clock phase properly. This is accompanied by a reduced inducibility of the Per2 gene in response to light. 